\title{
CMS: Physics overview
}

\author{
Vadim Alexakhin ${ }^{1, *}$ on behalf of the CMS Collaboration \\ ${ }^{1}$ Joint Institute for Nuclear Research, Dubna, Russia
}

\begin{abstract}
An overview of physics results from the CMS experiment at the LHC is given. The present analysis is based on data obtained for colliding proton beams at the c.m. energies of $\sqrt{s}=8$ and $13 \mathrm{TeV}$ over the period of LHC Run-1 and Run-2.

New unique data on interactions of Standard Model particles at record energies were obtained in the course of the first run of LHC operation. The Higgs boson was discovered, and investigation of its properties was initiated. Measurements were performed for Standard Model processes, including rare and one previously unobserved. This allowed one to refine some parameters of the Standard Model and set limits on the parameters of some theoretical models beyond the Standard Model, for example, on the masses of new particles, the fundamental energy scales, the coupling constants, and the cross sections for the new particles production.
\end{abstract}

\section{Introduction}

This report covers recent physics results from CMS [1] including precision measurements of the Higgs boson, first observed by the ATLAS and CMS collaborations in 2012 [2],[3] and top quark properties, b-physics, and Standard Model (SM) phenomena; searches for new particles. It will conclude with a summary and outlook.

In 2015, the Large Hadron Collider operated successfully for the first time at $13 \mathrm{TeV}$ and with $25 \mathrm{~ns}$ bunch spacing. In 2106-2018, the LHC concentrated on providing the experiments with very high integrated luminosity. This was achieved by the LHC's reaching a peak initial luminosity of $2 \times 10^{34} \mathrm{~cm}^{-2} \mathrm{~s}^{-1}$ and providing very high availability for physics.

The peak luminosity, corresponding to a number of simultaneous interactions per beam crossing (pileup) of about 40, exceeded the design goal for the LHC and CMS itself. In anticipation of a high peak and integrated luminosity in 2016 and beyond, CMS made various improvements and upgrades to the detector. These included the improved Level 1 Muon Trigger and Calorimeter Triggers. With these improvements, CMS was able to fully exploit the high integrated luminosity provided by the LHC.

In order to record the data efficiently with the high luminosities of 2016 and subsequent years, CMS implemented several innovative analysis techniques. Two of them are Particle Flow (PF) [4] and Pile UP per Proton Interaction (PUPPI) [5].

*e-mail: Vadim.Alexakhin@cern.ch 


\section{Recent Physics Results}

CMS has continued its prolific production of physics results. During the XXIVth International Baldin Seminar on High Energy Physics Problems 2018, 776 papers were submitted to peer reviewed journals. More than 25 new results were presented at the 2018 end-of-summer conferences. Here, we present a few highlights of recent results, many of which were shown during summer 2018.

\subsection{Higgs Boson Properties}

With more total luminosity and higher energy, larger samples of Higgs bosons are reconstructed, making more precise measurements and new studies possible.

Higgs decay to $\tau^{+} \tau^{-}$: The decay of the Higgs into $\tau^{+} \tau^{-}$is reconstructed in four separate decay modes, $e \mu, e \tau_{h}, \mu \tau_{h}$, and $\tau_{h} \tau_{h}$ and in three production channels, 0 -jet, Vector Boson Fusion (VBF), and "boosted" one [6]. Here $\tau_{h}$ refers to specific targeted hadronic decay modes. The data set corresponds to the integrated luminosity of $35.9 \mathrm{fb}^{-1}$. The $H \rightarrow \tau^{+} \tau^{-}$ signal is established with a significance of 4.9 standard deviations, to be compared to the expected significance of 4.7 standard deviations. The best fit of the product of the observed $H \rightarrow \tau^{+} \tau^{-}$signal production cross section and branching fraction is $1.09_{-0.26}^{+0.27}$ multiplied by the Standard Model expectation (Fig. 1, right). The combination with the corresponding measurement performed with the data collected by the CMS experiment at center-of-mass energies of 7 and $8 \mathrm{TeV}$ leads to the observed significance of 5.9 standard deviations, equal to the expected significance. This will constitute the first observation in a single experiment of coupling the Higgs to a fermion, a lepton, and the third generation family member. It also provides a validation of the Higgs Yukawa interaction.
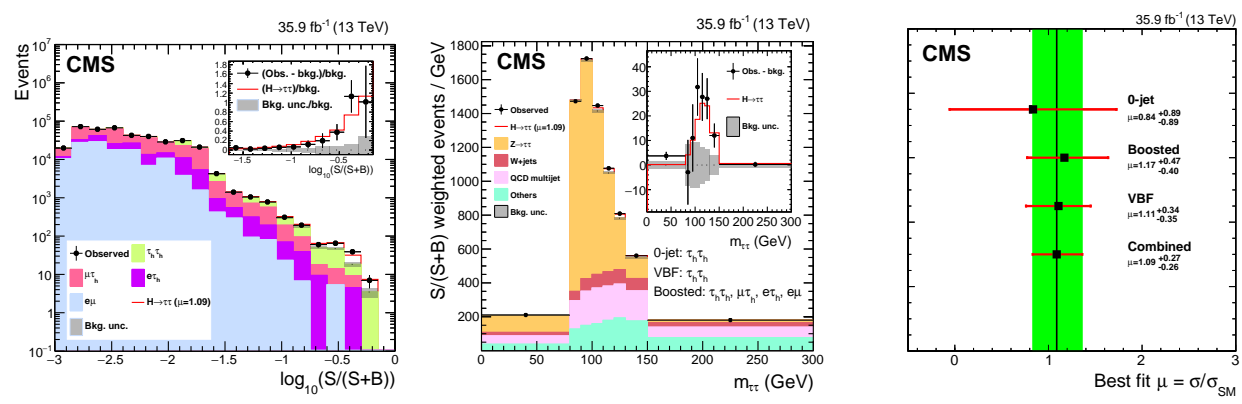

Figure 1. Distribution of the decimal logarithm of the ratio between the expected signal and the sum of the expected signal and expected background(left plot) [6]. Combined observed and predicted $m_{\tau \tau}$ distributions (center plot) [6]. Best fit signal strength per category (rigth plot) [6], for $m_{H}=125.09$ $\mathrm{GeV}$

$t \bar{t} H$ production: CMS has seen evidence for the $t \bar{t}$-Higgs coupling for Higgs bosons produced in conjunction with a top quark-antiquark pair and decaying to pairs of $\mathrm{W}$ bosons, $\mathrm{Z}$ bosons, photons, $\tau$ leptons, or bottom quark jets combined to maximize sensitivity [7].

An excess of events has been observed, with a significance of 5.2 standard deviations, over the expectation from the background-only hypothesis. The corresponding expected significance from the Standard Model for the Higgs boson mass of $125.09 \mathrm{GeV}$ is 4.2 standard deviations. The combined best fit signal strength normalized to the Standard Model prediction is $1.26_{-0.26}^{+0.31}$ (Fig. 2, right). 

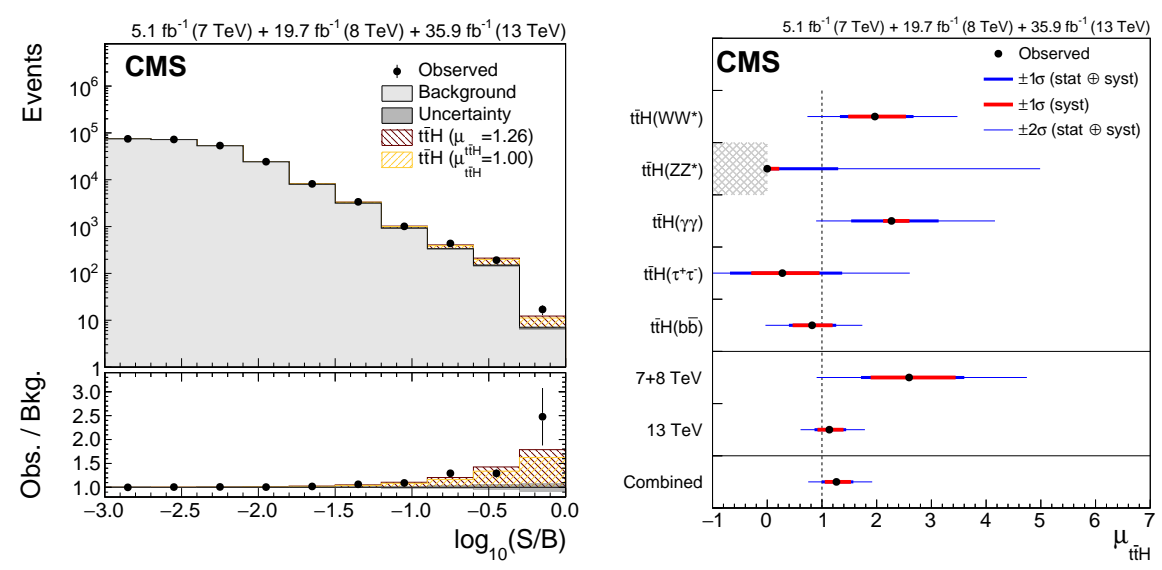

Figure 2. Left plot shows the distribution of events as a function of the decimal logarithm of $S / B$ [7]. Right plot: Best fit value of the $t \bar{t} H$ signal strength modifier $\mu_{t \bar{t} H}$ [7]

Higgs boson decay to $b \bar{b}$ : Recently CMS has made an observation of the Higgs boson decay to bottom quarks [8]. The measurement examines the $V H$ production process, where the Higgs boson is produced in association with a $W$ or $Z$ boson and decays into $b \bar{b}$. The data comprise proton-proton $(p p)$ collisions recorded at $\sqrt{s}=13 \mathrm{TeV}$ corresponding to the total integrated luminosity of $41.3 \mathrm{fb}^{-1}$. Higgs boson candidates have been reconstructed from the pair of jets in the event most likely to originate from $b$ quarks.
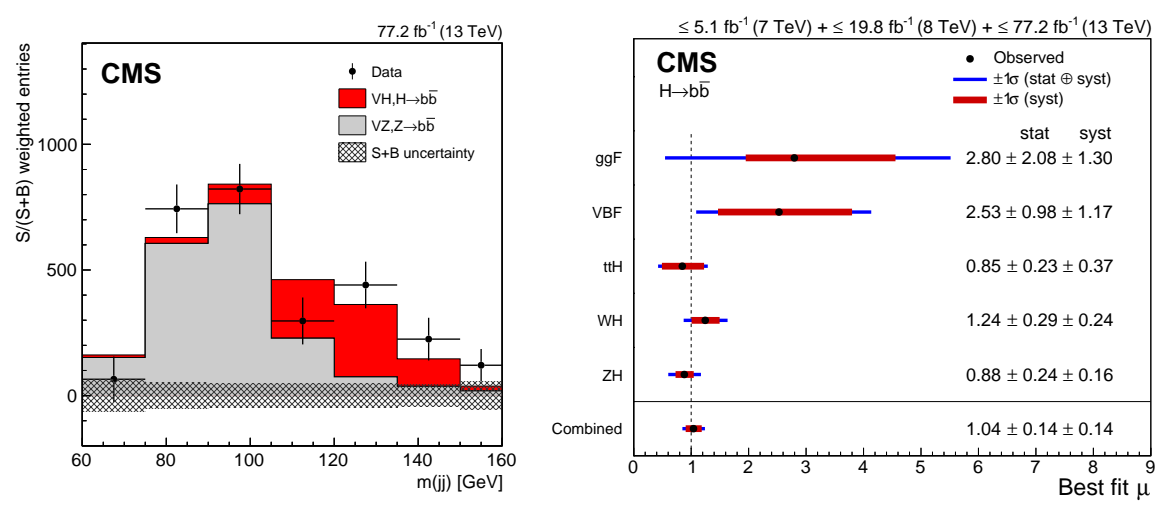

Figure 3. Left plot: dijet invariant mass distribution for events weighted by $S /(S+B)$ (left plot) [8]. Right plot: best-fit value of the $H \rightarrow b \bar{b}$ signal strength for the five individual production modes considered [8]

A combination of all CMS measurements of the $V H, H \rightarrow b \bar{b}$ process using the protonproton collisions recorded at the center of mass energies of 7,8 , and $13 \mathrm{TeV}$, yields the observed (expected) significance of 4.8 (4.9) standard deviations at $m_{H}=125.09 \mathrm{GeV}$, and the signal strength is $\mu=1.01 \pm 0.22$. Combining this result with previous measurements by the CMS Collaboration of the $H \rightarrow b \bar{b}$ decay in events where the Higgs boson is produced through gluon fusion, vector boson fusion, or in association with top quarks, the observed (expected) significance increases to 5.6(5.5) standard deviations and the signal 
strength is $\mu=1.04 \pm 0.20$. This constitutes the observation of the $H \rightarrow b \bar{b}$ decay by the CMS Collaboration. All results are summarized in Fig. 3, right plot.

Higgs boson decay to $\mu \mu$ : The paper [10] presents a search for the Higgs boson decaying to two muons using the data recorded by the CMS experiment at the LHC in 2016 at a centerof-mass energy of $13 \mathrm{TeV}$, corresponding to the integrated luminosity of $35.9 \mathrm{fb}^{-1}$.

No significant evidence for the decay $H \rightarrow \mu^{+} \mu$ - has been observed. Limits are set on the cross section multiplied by the branching fraction of the Higgs boson decaying to two muons. The combination with the data recorded at center-of-mass energies of 7 and $8 \mathrm{TeV}$ yields a $95 \%$ confidence level observed upper limit of 2.92 multiplied by the Standard Model value for $m_{H}=125.09 \mathrm{GeV}$. The corresponding expected upper limit in the absence of a SM decay in this channel is 2.16, which is the most sensitive to date. Assuming the Standard Model production cross sections for the Higgs boson, the observed limit corresponds to an upper limit of $6.4 \times 10^{-4}$ on the Higgs boson branching fraction to two muons.

Higgs combination: A set of combined measurements of Higgs boson production and decay rates has been presented by CMS in work [11], along with the consequential constraints placed on its couplings to Standard Model (SM) particles, and on the parameter spaces of several Beyond the Standard Model (BSM) scenarios. The combination is based on analyses targeting the gluon fusion and vector boson fusion production modes, and associated production with a vector boson or a pair of top quarks. The analyses included in the combination target Higgs boson decay in the $H \rightarrow Z Z, W W, \gamma \gamma, \tau \tau, b \bar{b}$, and $\mu \mu$ channels, using $13 \mathrm{TeV}$ proton-proton collision data collected in 2016 and corresponding to an integrated luminosity of $35.9 \mathrm{fb}^{-1}$. The per-production and per-decay mode signal strength modifiers are presented in Fig. 4. Additionally, searches for invisible Higgs boson decays are included to increase the sensitivity to potential interactions with BSM particles.
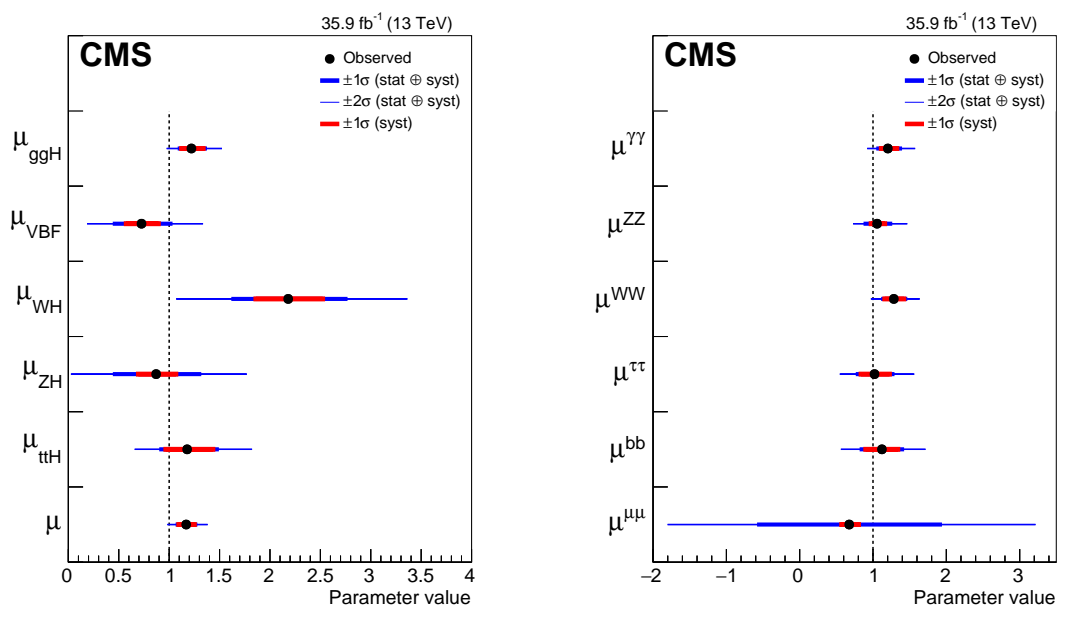

Figure 4. Summary plot of the fit to the per-production mode (left) [11] and per-decay mode (right) signal strength modifiers [11]

Measurements of the Higgs boson production cross section multiplied by the branching fraction in each of the channels are presented, along with generic parametrization in terms of ratios of production cross sections and branching fractions, that makes no assumptions about the Higgs boson total width. The combined signal yield relative to the SM prediction has been measured as $1.17 \pm 0.10$ at $m_{H}=125.09 \mathrm{GeV}$. An improvement in the measured precision of 
the gluon fusion production rate of around $\sim 50 \%$ is achieved compared to previous ATLAS and CMS measurements.

\subsection{Top Quark Properties}

The top quark $t$ is the heaviest Standard Model particle with the largest Yukawa coupling to the Higgs. As physics beyond the Standard Model is generally expected at higher scales, the top quark is the particle with the smallest mass gap to any other not yet discovered new particle and is often expected to be produced in association with new physics, such as supersymmetry. The precise knowledge of Standard Model top quark processes is essential, as it is an important background for search analyses but also because it provides through Standard Model precision fits direct constraints on new physics.

Top quark cross sections: The cross section for $t-\bar{t}$ production as a function of center of mass energy is shown in Fig. 5, left plot. The result for $13 \mathrm{TeV}$ from CMS [12] is $833 \pm 33 \mathrm{pb}$, in good agreement with theory. Summary of ATLAS and CMS measurements of the single top quark production cross-sections in various channels as a function of the center of mass energy is shown in Fig. 5, right plot.

Single and double differential cross sections for key variables of top quark pair production are now also measured. In work [13] measurements are presented of absolute and normalized differential cross sections of $t \bar{t}$ production based on both particle-level objects in a fiducial phase space and parton-level top quarks in a full phase space using pp collisions at a centreof-mass energy of $13 \mathrm{TeV}$ recorded by the CMS detector. Most of themeasured differential cross sections are well modeled by theoretical predictions. However, significant disagreement between the data and next-to-leading-order Monte Carlo is observed for the transverse momentum of top quarks, leptons, b jets, and $t \bar{t}, l \bar{l}$ and $b \bar{b}$ systems and for the invariant mass of the $t \bar{t}, l \bar{l}$ and $b \bar{b}$ systems. Similar levels of disagreement are observed for predictions with beyond NLO precision.
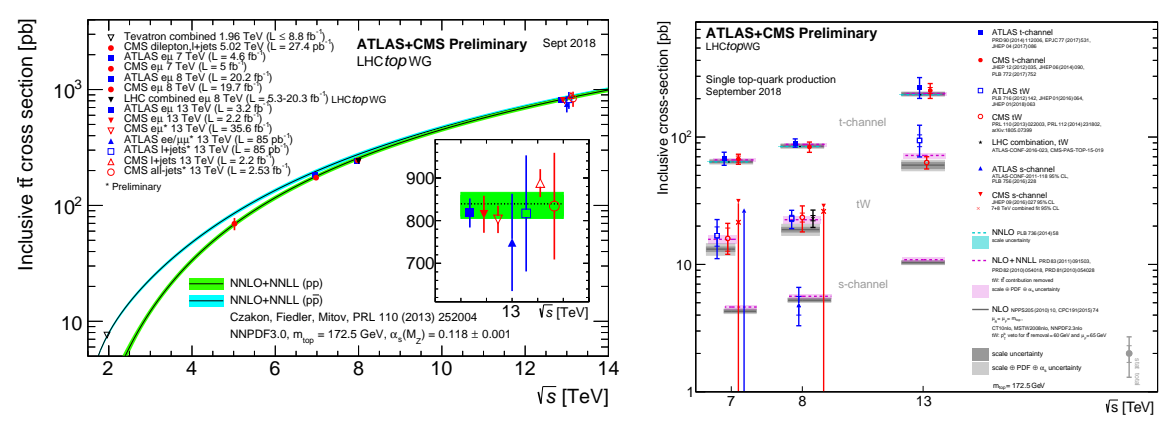

Figure 5. Left plot: top quark pair production total cross section vs. center of mass energy [12]. Right plot: summary of ATLAS and CMS measurements of the single top quark production cross-sections in various channels as a function of the center of mass energy [12]

The cross sections for the production of single top quarks and antiquarks in the t-channel, and their ratio, were measured in proton-proton collisions at a center-of-mass energy of $13 \mathrm{TeV}$ using integrated luminosity of $35.9 \mathrm{fb}^{-1}$ [14]. Events with one muon or electron and two jets have been selected, where one of the two jets is identified as originating from a bottom quark. The ratio Rt-ch. of the cross sections (see Fig. 6, left plot) has been measured to be $1.65 \pm 0.02$ (stat) \pm 0.04 (syst), what is in agreement with the SM predictions. 
Top quark rare decays: Fig. 6, right plot, shows recently published results on rare flavorchanging neutral current decays of top quark [15]. While measurements at the LHC will not reach the level predicted by the SM, for some decays we will soon approach the predictions of various models of new physics and will be able to confirm them or rule them out.
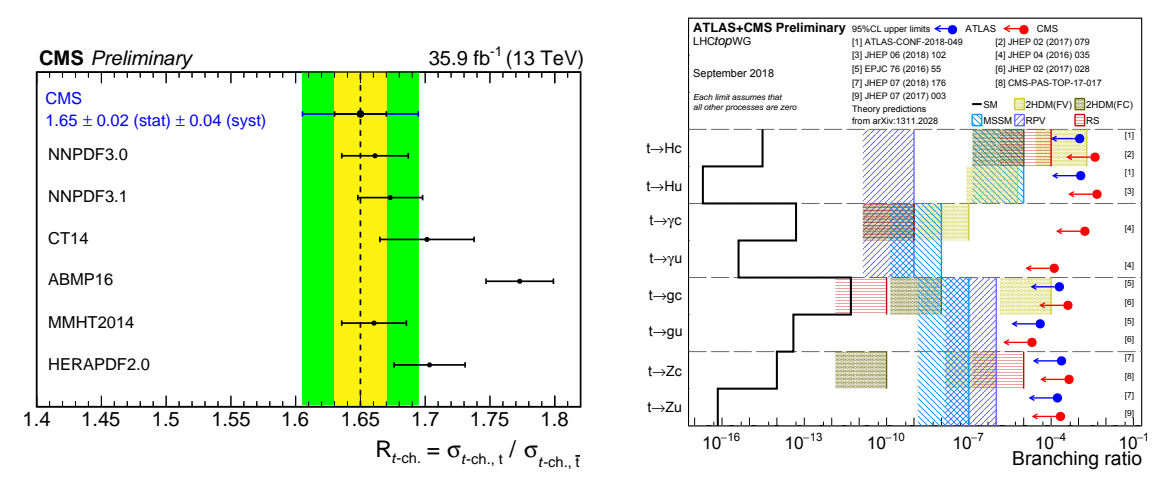

Figure 6. Left plot: Comparison of the measured Rt-ch. (dotted line) with the prediction from different PDF sets [14]. Right plot: limits on rare decays of the top quark [15]

Top quark gallery: The first evidence of events consistent with the production of a single top quark in association with a photon was reported in work [16]. The measured product of the cross section and branching fraction is $\sigma(p p \rightarrow t \gamma \mathrm{j}) \mathcal{B}(t \rightarrow \gamma v b)=115 \pm$ 17 (stat) \pm 30 (syst) fb, which is consistent with the Standard Model prediction.

The first observation of top quark production in proton-nucleus collisions is reported using proton-lead data collected by the CMS experiment [17] The measured cross section is $\sigma_{t \bar{t}}=45 \pm 8 \mathrm{nb}$, consistent with the predictions from perturbative quantum chromodynamics. standard deviations.

\subsection{B physics}

The angular distribution of the flavor-changing neutral current decay $B^{+} \rightarrow K^{+} \mu^{+} \mu^{-}$was studied in proton-proton collisions at a center-of-mass energy of $8 \mathrm{TeV}$ [18]. The analysis is based on the data corresponding to an integrated luminosity of $20.5 \mathrm{fb}^{-1}$. The forwardbackward asymmetry $A_{F B}$ of the dimuon system and the contribution $F_{H}$ from the pseudoscalar, scalar, and tensor amplitudes to the decay width have been measured as a function of the dimuon mass squared $\left(q^{2}\right)$. The measurements are consistent with the Standard Model expectations.

Data samples of $p p$ collisions at $\sqrt{s}=13 \mathrm{TeV}$, collected by CMS in the years 2015-2017, corresponding to an integrated luminosity of $80.0 \mathrm{fb}^{-1}$, were used to measure the invariant mass distribution of the $\chi_{b}(3 P) \rightarrow \Upsilon(3 S) \gamma$ candidates (see Fig. 7, left plot), with the $\Upsilon(3 S)$ mesons detected in the dimuon decay channel and the photons reconstructed through conversions to $e e$ pairs [19]. The measured distribution is well reproduced by the superposition of the $\chi_{b 1}(3 P)$ and $\chi_{b 2}(3 P)$ quarkonium states, overlaid on a smooth continuum (see Fig. 7 , right plot). This is the first time that the two states have been individually observed. Their mass difference is $\Delta M=10.60 \pm 0.64$ (stat) \pm 0.17 (syst) $\mathrm{MeV}$, This measurement fills a gap in the spin-dependent bottomonium spectrum below the open-beauty threshold and should significantly contribute to improved understanding of the nonperturbative spin-orbit interactions affecting quarkonium spectroscopy. 

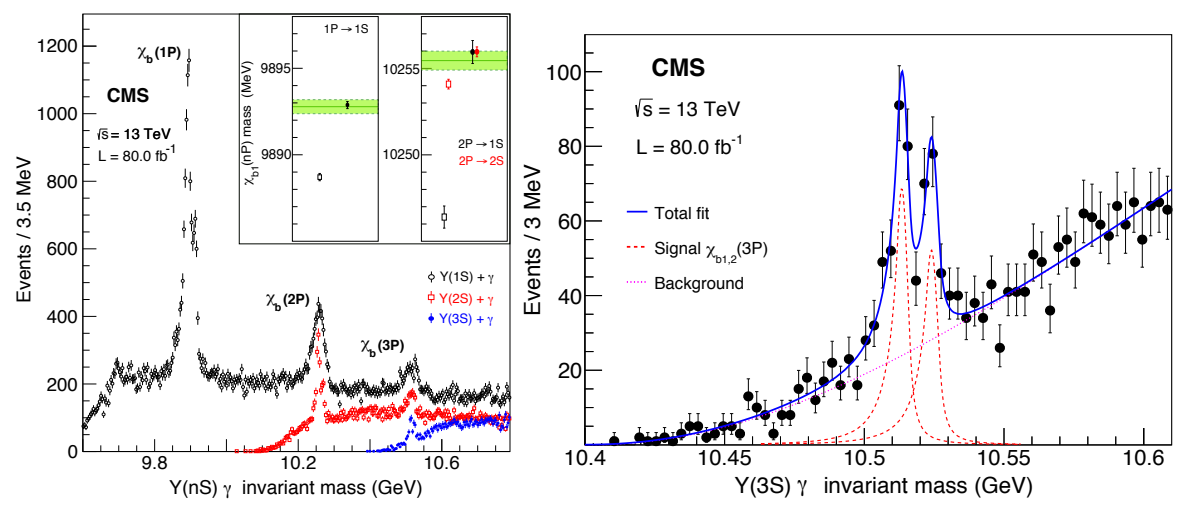

Figure 7. Left plot: the invariant mass distributions of the $\chi_{b J} \rightarrow \Upsilon(n S) \gamma$ candidates $(n=1,2,3)$. The inset shows the $\chi_{b 1}(1 P)$ and $\chi_{b 1}(2 P)$ masses. The world-average values are shown by the horizontal bands, with dashed lines representing their total uncertainties [19]. Right plot: the invariant mass distribution of the $\chi_{b}(3 P) \rightarrow \Upsilon(3 S) \gamma$ candidates [19]

\subsection{Searches for New Particles}

Black Holes: A search in energetic, high-multiplicity final states for evidence of physics beyond the Standard Model, such as black holes, string balls, and electroweak sphalerons, is presented in work [20]. In the channel with high-multiplicity the final state semiclassical black holes with minimum masses as high as $10.1 \mathrm{TeV}$ and string balls with masses as high as $9.5 \mathrm{TeV}$ are excluded by this search.

Extra gauge bosons: A search for narrow resonances in dielectron and dimuon invariant mass spectra has been performed using the data recorded in 2016 from proton-proton collisions at $\sqrt{s}=13 \mathrm{TeV}$ [21]. Observations are in agreement with Standard Model expectations (see Fig. 8). The upper limits at $95 \%$ confidence level on the product of a narrow-resonance production cross section and branching fraction to dileptons have been calculated in a modelindependent manner to enable interpretation in the framework of the models predicting a narrow dielectron or dimuon resonance.

Limits are set on the masses of various hypothetical particles. For the $Z_{\text {SSM }}^{\prime}$ particle, which arises in the sequential Standard Model, and for the superstring-inspired $Z_{\psi}^{\prime}$ particle, $95 \%$ confidence level lower mass limits for the combined channels are found to be 4.5 and $3.9 \mathrm{TeV}$, respectively. The corresponding limits for Kaluza-Klein gravitons arising in the Randall-Sundrum model of extra dimensions with coupling parameters $k / \bar{M}_{\mathrm{Pl}}$ of $0.01,0.05$, and 0.10 are $2.1,3.65$, and $4.25 \mathrm{TeV}$, respectively.

\section{Search for low-mass Resonances}

A search for resonances in the mass range $12-70 \mathrm{GeV}$ produced in association with a $b$ quark jet and a second jet, and decaying to a muon pair, is reported in work [22]. The analysis is based on data from proton-proton collisions at center-of-mass energies of 8 and $13 \mathrm{TeV}$ corresponding to integrated luminosities of 19.7 and $35.9 \mathrm{fb}^{-1}$, respectively. The search has been carried out in two mutually exclusive event categories. Events in the first category (SR1) are required to have a $b$ quark jet in the central region $(|\eta| \leq 2.4)$ and at least one jet in the forward region $(|\eta|>2.4)$. Events in the second category (SR2) are required to have two jets in the central region, at least one of them is identified as a $b$ quark jet, no jets in the forward region, and low missing transverse momentum. An excess of events above the background near a dimuon mass of $28 \mathrm{GeV}$ has been observed in the $8 \mathrm{TeV}$ data, 

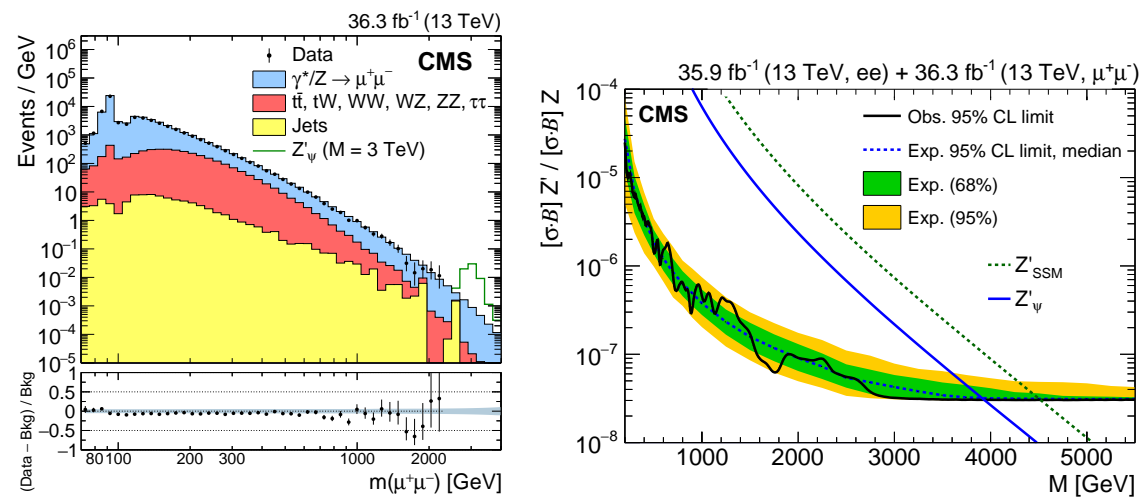

Figure 8. Left plot: the invariant mass spectra of dimuon events. The histograms represent the expectations from the SM processes. Example signal shapes for a narrow resonance with a mass of $3 \mathrm{TeV}$ are shown by the stacked open histograms [21]. Right plot: the upper limits at 95\% CL on the product of production cross section and branching fraction for a spin-1 resonance with a width equal to $0.6 \%$ of the resonance mass, relative to the product of production cross section and branching fraction of a $Z^{\prime}$ boson, for combination of dielectron and dimuon channels. Theoretical predictions for the spin-1 $Z_{\mathrm{SSM}}^{\prime}$ and $Z_{\psi}^{\prime}$ resonances are shown for comparison [21]

corresponding to local significances of 4.2 and 2.9 standard deviations for the first and second event categories, respectively (see Fig. 9). A similar analysis conducted with the $13 \mathrm{TeV}$ data results in a mild excess over the background in the first event category corresponding to a local significance of 2.0 standard deviations, while the second category results in a 1.4 standard deviation deficit. In the lack of a realistic signal model, the $13 \mathrm{TeV}$ results are not sufficient to make a definitive statement about the origin of the $8 \mathrm{TeV}$ excess. Therefore, more data and additional theoretical input are both required to fully understand the results presented in this work.
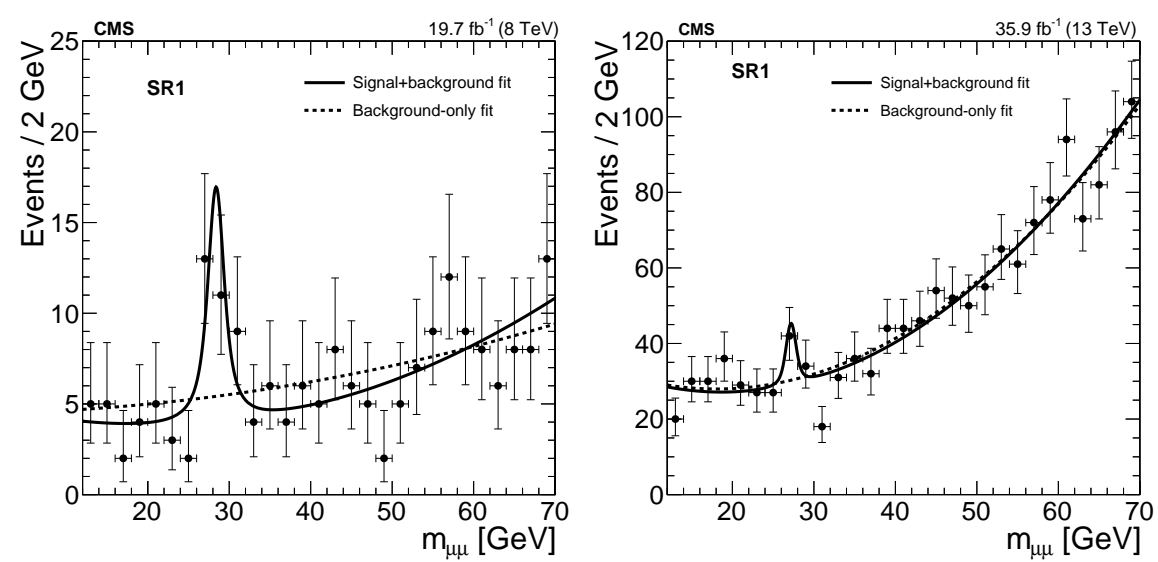

Figure 9. The $12<m_{\mu \mu}<70 \mathrm{GeV}$ range for $\mathrm{SR} 1$ in the $8 \mathrm{TeV}$ (left) and $13 \mathrm{TeV}$ (right) analysis [22]

Supersymmetry: Supersymmetry (SUSY) is an extension of the SM that resolves some of its open issues: the hierarchy problem, the unification of the couplings of strong and EWK 
forces, and the provision of a candidate for dark matter. Many physicists expected SUSY to be seen soon after LHC startup, with as little as $100 \mathrm{pb}^{-1}$ of luminosity, and to be the first major LHC discovery -- before even the Higgs boson.

Now with more than $90 \mathrm{fb}^{-1}$ of integrated luminosity at $13 \mathrm{TeV}$, SUSY has not yet been observed. The reasons for this could be: the lightest SUSY particles may be heavier than we thought; the pattern of decays may be more devious/obscure than we thought (coverage for R-Parity violating SUSY and long-lived particles are not as complete as for MSSM or PMSSM searches); maybe SUSY does not cure all the above problems and there are several new physics scenarios, including SUSY, forming a complicated landscape in which we are currently lost; or maybe SUSY is just another promising idea that nature does not choose to follow.

CMS continues to pursue a broad program of SUSY searches. Searches investigate different final states and use several complementary analysis techniques [23].

Long-lived particles: Many BSM models predict long-lived particles. These result in distinctive properties such as displaced vertices, displaced conversions, displaced photons or jets, displaced leptons or dileptons, heavy stable charged particles (HSCP) with very high ionization losses, or stopped HSCP. Some of these can be observed by special searches, usually with special triggers. A recent example is a search by CMS for stopped long-lived particles. The signature is a high energy jet in the calorimeter that is out of time with collisions. This search excluded gluinos with lifetimes from $10 \mu \mathrm{s}$ to $1000 \mathrm{~s}$ for gluino masses $<1379 \mathrm{GeV}$; and top squarks in this lifetime range with stop mass $<740 \mathrm{GeV}[24]$. This is an exciting new area of inquiry that has not been thoroughly explored at the LHC.

\section{Conclusions}

The outstanding performance of the Large Hadron Collider and the CMS detector have made it possible to collect a $13 \mathrm{TeV}$ data set corresponding to an integrated luminosity of $90 \mathrm{fb}^{-1}$ in 2016-2017. The most recent result highlights derived by using this data have been presented here. So far, only of the order of 5 percent of the total expected luminosity during the lifetime of the LHC has been recorded. The data that is yet to come will allow for many more precision measurements and direct or indirect searches for new physics.

The author wishes to thank the many members of the CMS Collaboration and their technical and administrative staffs whose efforts are summarized in this report. We congratulate our colleagues in the CERN accelerator departments for the excellent performance of the LHC.

\section{References}

[1] CMS Collaboration, JINST 3, S08004 (2008)

[2] G. Aad et al. [ATLAS Collaboration], Phys. Lett. B 716, 1 (2012)

[3] S. Chatrchyan et al. [CMS Collaboration], Phys. Lett. B 716, 30 (2012)

[4] CMS Collaboration, Particle-flow reconstruction and global event description with the CMS detector, arXiv:1706.04965, CMS-PRF-14-001, CERN-EP-2017-110

[5] D. Bertolini, P. Harris, M. Low, and N. Tran, JHEP 10, 059 (2014)

[6] CMS Collaboration, Physics Letters B 779, 283-316 (2018)

[7] CMS Collaboration, Phys. Rev. Lett. 120, 231801 (2018)

[8] CMS Collaboration, Phys. Rev. Lett. 121, 121801 (2018)

[9] CMS Collaboration, JHEP 09, 087 (2014) 
[10] CMS Collaboration, Search for the Higgs boson decaying to two muons in protonproton collisions at $\sqrt{s}=13 \mathrm{TeV}$, CMS-HIG-17-019, CERN-EP-2018-165

[11] CMS Collaboration, Combined measurements of Higgs boson couplings in protonproton collisions at $\sqrt{s}=13 \mathrm{TeV}$, CMS-HIG-17-031, CERN-EP-2018-263

[12] LHC Top Physics Working Group, http://twiki.cern.ch/twiki/bin/view/LHCPhysics/LHCTopWGSummaryPlots

[13] CMS Collaboration, Measurements of differential cross sections for $t \bar{t}$ production in proton-proton collisions at $\sqrt{s}=13 \mathrm{TeV}$ using events containing two leptons, CMS-PASTOP-17-014

[14] CMS Collaboration, Measurement of the single top quark and antiquark production cross sections in the thannel and their ratio pp collisions at $\sqrt{s}=13 \mathrm{TeV}$, CMS-PASTOP-17-011

[15] CMS Collaboration, Summary of FCNC upper limits at the $95 \%$ CL from CMS searches at $8 \mathrm{TeV}$, http://twiki.cern.ch/twiki/bin/view/CMSPublic/PhysicsResultsTOPSummaryFigures

[16] CMS Collaboration, Evidence for the associated production of a single top quark and a photon in proton-proton collisions at $\sqrt{s}=13 \mathrm{TeV}$, CMS-TOP-17-016, CERN-EP-2018206

[17] CMS Collaboration, Phys. Rev. Lett. 119, 242001 (2017)

[18] CMS Collaboration, Angular analysis of the decay $B^{+} \rightarrow K^{+} \mu^{+} \mu^{-}$in proton-proton collisions at $\sqrt{s}=8 \mathrm{TeV}$, CMS-BPH-15-001, CERN-EP-2018-125

[19] CMS Collaboration, Phys. Rev. Lett. 121, 092002 (2018)

[20] CMS Collaboration, Search for black holes and sphalerons in high-multiplicity final states in proton-proton collisions at $\sqrt{s}=13 \mathrm{TeV}$, CMS-EXO-17-023, CERN-EP-2018093

[21] CMS Collaboration, JHEP 06, 120 (2018)

[22] CMS Collaboration, Search for resonances in the mass spectrum of muon pairs produced in association with $b$ quark jets in proton-proton collisions at $\sqrt{s}=8$ and $13 \mathrm{TeV}$, CMS-HIG-16-017, CERN-EP-2018-204

[23] CMS Collaboration, CMS Supersymmetry Physics Results, https://twiki.cern.ch/twiki/bin/view/CMSPublic/SUSYSummary2017

[24] CMS Collaboration, JHEP 05127 (2018) 